\title{
Analysis on the Clinical Therapeutic Effect of Hepatic Artery Chemoembolization in the Treatment of Advanced Liver Cancer
}

Wei Zheng, Huilin Chang, Xin Chen ${ }^{*}$

Shaanxi Provincial People's Hospital, Xi'an 710068, Shaanxi Province, China

\begin{abstract}
Objective: To analyze the clinical efficacy of hepatic artery chemoembolization in the treatment of advanced liver cancer. Methods: 124 patients with advanced liver cancer admitted to our hospital from September 2019 to November 2020 were selected as the research subjects of this paper. The patients with advanced liver cancer were divided into experimental group and control group. The control group was treated with radiofrequency ablation alone, and the experimental group was administered hepatic arterial

control group, and the difference in the remission rate between the two groups of patients was statistically significant. Conclusion: The implementation of hepatic arterial chemoembolization for patients with advanced liver cancer can promote the improvement of the patient's short-term treatment efficacy, enhance the liver functions of the patient, reduce the incidence of adverse reactions, improve the efficiency of the patient's body rehabilitation, and enhance the quality of life of the patient after treatment.
\end{abstract} chemoembolization. The improvement in physical indicators and the incidence of adverse reactions between the two groups were compared. Results: The alpha-fetoprotein (AFP) index and serum total bilirubin (TBIL) index of the experimental group were lower than those of the control group, and the alanine aminotransferase (ALT) index was higher than that of the control group. There were differences in the comparison of liver function indices between the two groups which were statistically significant. After treatment, there were 3 cases of fever, 4 cases of vomiting, 8 cases of bone marrow transplantation, 4 cases of abdominal pain, 2 cases of proteinuria, and 1 case of diarrhea occurred in the experimental group; whereas there were 6 cases of fever, 8 cases of vomiting, 14 cases of bone marrow transplantation, 7 cases of abdominal pain, 5 cases of proteinuria, and 6 cases of diarrhea occurred in the control group. The difference in incidence of adverse reactions between patients after different treatment interventions was statistically significant. Analyzing the remission rate of tumor diseases in patients, the remission rate of the experimental group was higher than that of the

Key words: Hepatic artery chemoembolization; Advanced liver cancer; Treatment efficacy; Incidence of adverse reaction

\author{
Publication date: March, 2021 \\ Publication online: 31 March, 2021 \\ *Corresponding author: Xin Chen, zhengwei03168@163. \\ com
}

Primary liver cancers are common clinical malignancies with very high incidence, fatality and recurrence rates. More than $90 \%$ of patients with liver cancer are hepatocellular carcinoma, and the best treatment option is surgical resection. Surgical treatment is affected by factors such as hidden cancer lesions and nodularity, and some patients are accompanied by other diseases. Therefore, the patient has been diagnosed in advanced stage and missed the best treatment time. Patients who do not meet the indications for surgical treatment need to carry out hepatic artery chemoembolization, radiofrequency ablation, radiotherapy, targeted therapy and other treatment methods. Hepatic artery chemoembolization is a common interventional procedure. This paper 
analyzes the therapeutic effects of hepatic artery chemoembolization and radiofrequency ablation for patients with advanced liver cancer.

\section{General information and methods}

\subsection{General information}

124 patients with advanced liver cancer admitted to our hospital from September 2019 to November 2020 were selected as the research subjects of this paper. The patients with advanced liver cancer were divided into experimental group and control group, with 62 patients in each group. There were 42 males and 20 females in the experimental group. The average age of the patients was $(55.82 \pm 5.36)$ years old, and the average tumor diameter was $(4.13 \pm 0.35) \mathrm{cm}$; there were 40 males and 22 females in the control group, and the average age of the patients was $(55.41 \pm 5.23)$ years old, average tumor diameter was $(4.15 \pm 0.36) \mathrm{cm}$. Inclusion criteria: Patients have a certain understanding on treatment methods, volunteer to participate in the experiment in this paper, and the case information are complete; patients were diagnosed with advanced lung cancer by B-ultrasound, CT, MRI, AFP, liver biopsy and other examination results. Exclusion criteria: patients with diffuse liver cancer; patients with severe organ dysfunction diseases; patients with psychiatric diseases were not included in this experimen ${ }^{\mathrm{t}[1]}$.

\subsection{Methods}

\subsubsection{Hepatic artery channelization therapy}

After carrying out routine disinfection and draping for the patient, percutaneous femoral artery puncture with Seldinger method was performed. After inserting the catheter sheath, the imaging system was followed through DSA, the main blood supply artery of the lesion was selected, and contrast examination was performed after inserting the catheter to observe the arteries and lesions and the distribution of blood vessels. Then, drug was inserted through the catheter. The chemotherapy drugs selected were pirarubicin 20-30mg, cisplatin $80 \mathrm{mg}$, iodized oil $5-10 \mathrm{ml}$ and epirubicin $20 \mathrm{mg}^{[2]}$. Then gelatin sponge was used for adjuvant embolization at the end of the artery. After treatment, the blood supply of the patient's lesion was observed, and hepatic artery chemoembolization was carried out every one month or $\mathrm{so}^{[3]}$.

\subsubsection{Radiofrequency ablation therapy}

CT examination was performed on the patients, and the location of the lesion was determined according to the examination results. The puncture point and puncture path were determined in advance, the lesions were punctured with radiofrequency electroneedle, and then ablated to cover the lesions and surrounding normal tissues, and the radiofrequency parameters were adjusted according to the patient's conditions ${ }^{[4]}$.

\subsection{Assessment criteria}

The disappearance of the tumor lesions in the patient indicates complete remission; the elimination of the lesion area in the patient more than $50 \%$ indicates partial remission; the elimination of the tumor area in the patient less than 50\% indicates stable; an increase in tumor area of more than $25 \%$ indicates progression. The remission rate is the total percentage of complete remission and partial remission ${ }^{[5]}$.

\subsection{Statistical method}

Using SPSS24.0 statistical software for data analysis in this paper, the patient's disease remission rate and incidence of adverse reaction are all count data, checked by $\chi^{2}$ test, expressed in $\%$; the improvement level of patients' liver function index was checked by t-test, expressed in \%. Differences in treatment effects and improvement in indicators between the two groups of patients indicate statistical significance.

\section{Results}

\subsection{Comparison on the remission rate between the two groups of patients}

Analyzing the remission rate of tumor diseases in patients, the remission rate of the experimental group was higher than that of the control group, and the difference in the remission rate of the two groups of patients was statistically significant. See Table 1.

Table 1. Comparison on the remission rate between the two groups of patients

\begin{tabular}{ccccccc}
\hline Group & No. of Cases & Complete Remission & Partial Remission & Stable & Progression & Remission Rate (\%) \\
\hline Experimental & 62 & 48 & 8 & 5 & 1 & $56(90.32 \%)$ \\
Control & 62 & 35 & 5 & 11 & 11 & $40(64.52 \%)$ \\
$P$ & & & & & $<0.05$ \\
\hline
\end{tabular}




\subsection{Comparison of liver function indices between} the two groups of patients

The alpha-fetoprotein (AFP) index and serum total bilirubin (TBIL) index of the experimental group were lower than those of the control group, and the alanine aminotransferase (ALT) index was higher than that of the control group. There was a difference in the liver function indices between the two groups, with statistical significance. See Table 2.

Table 2. Comparison of liver function indices between the two groups of patients

\begin{tabular}{ccccc}
\hline Group & & AFP $\left(\mathbf{n g} \cdot \mathbf{m l}^{-1}\right)$ & TBIL $\left(\boldsymbol{\mu m o l} / \mathbf{L}^{-1}\right)$ & ALT $\left(\mathbf{u} \cdot \mathbf{L}^{-1}\right)$ \\
\hline \multirow{2}{*}{ Experimental } & Before Treatment & $211.25 \pm 15.26$ & $53.69 \pm 3.92$ & $25.82 \pm 1.56$ \\
& After Treatment & $75.21 \pm 5.36$ & $24 . .85 \pm 3.36$ & $52.69 \pm 3.65$ \\
Control & Before Treatment & $215.14 \pm 16.36$ & $54.21 \pm 4.28$ & $23.33 \pm 2.36$ \\
& After Treatment & $131.25 \pm 14.15$ & $35.36 \pm 3.65$ & $43.15 \pm 3.58$ \\
$P$ & & $<0.05$ & $<0.05$ & $<0.05$ \\
\hline
\end{tabular}

2.3 Comparison on the incidence of adverse reactions between the two groups of patients after treatment

After treatment, there were 2 cases of fever, 2 cases of vomiting, 6 cases of bone marrow transplantation, 1 case of abdominal pain, 2 cases of proteinuria, and 1 case of diarrhea in the experimental group; whereas there were 5 cases of fever, 4 cases of vomiting, and 8 cases of bone marrow transplantation, 4 cases of abdominal pain, 4 cases of proteinuria, and 3 cases of diarrhea in the control group, the difference in the incidence of adverse reaction in patients after different treatment interventions indicates statistical significance. See Table 3.

Table 3. Comparison on the incidence of adverse reactions between the two groups of patients after treatment

\begin{tabular}{ccccccccc}
\hline Group & No. of Cases & Fever & Vomiting & $\begin{array}{c}\text { Bone Marrow } \\
\text { Transplantation }\end{array}$ & Abdominal Pain Proteinuria & Diarrhea & Incidence (\%) \\
\hline Experimental & 62 & 2 & 2 & 6 & 1 & 2 & 1 & $14(22.58 \%)$ \\
Control & 62 & 5 & 4 & 8 & 4 & 4 & 3 & $28(45.16 \%)$ \\
$P$ & & & & & & & $<0.05$ \\
\hline
\end{tabular}

\section{Discussions}

Advanced liver cancer is a common clinical malignant tumor disease. Surgery, microwave ablation, hepatic artery chemoembolization, and traditional Chinese medicine treatment are all commonly used clinical modalities. The implementation of hepatic artery embolization for patients has extremely high therapeutic effects. Aidi injection is a new type of clinical anti-cancer injection which incorporates astragalus, acanthopanax bark, cantharidin and other drugs with the effects of promoting blood circulation, removing blood stasis, clearing heat and detoxifying, and ginseng polysaccharides also have anti-cancer effects which improve the immunity of patients ${ }^{[6]}$. Aidi injection also has the effect of inhibiting advanced liver cancer. Through hepatic artery chemoembolization, it can alleviate the patient's conditions and effectively prolong the patient's survival time. Reasonable treatment can reduce the incidence of adverse reaction and has high treatment safety $^{[7]}$.
In summary, the implementation of hepatic artery chemoembolization for patients with advanced liver cancer can promote the improvement of patients' short-term treatment efficacy, enhance patients' liver function indices, reduce the incidence of adverse reactions, improve the efficiency of patients' body rehabilitation, and enhance the quality of life of patients after treatment.

\section{References}

[1] Wu PS, Luo YX, Chen SH. Clinical efficacy and safety of different doses of apatinib combined with transarterial chemoembolization in advanced primary hepatocellular carcinoma [J]. Guangxi Medical Journal, 2020, 42(3):293-296, 301.

[2] Zhou YY, Ye GW, Xu YY. Analysis of short-term and longterm efficacy of elemene combined with TACE interventional therapy in patients with advanced liver cancer [J]. China Modern Doctor, 2020, 58(19):89-92.

[3] Ping F. Effect of transcatheter arterial chemoembolization combined with sorafenib in hepatocellular carcinoma patients in middle or advanced stage and nursing measures [J]. Journal 
of Clinical Medicine in Practice, 2020, 24(4):122-124,128.

[4] Zhu HG, Chen QL. Observations on the efficacy of simple hepatic artery chemoembolization combined with radiofrequency ablation in the treatment of advanced liver cancer [J].Clinical Research, 2021, 29(1):71-72.

[5] Lin XQ. Effects of hepatic artery chemoembolization combined with small-molecule targeted therapy on advanced liver cancer [J]. Shenzhen Journal of Integrated Traditional Chinese and Western Medicine, 2020, 30(1):184-185.
[6] You SW, Lin X, Hu YR, et al. Transarterial chemoembolization combined with laparoscopic microwave ablation for advanced hepatocellular carcinoma [J]. Journal of Hepatopancreatobiliary Surgery, 2020, 32(9):526-531.

[7] Huang JS, Zeng L, Cai JY. Observation on the effects of Aidi injection combined with transhepatic arterial chemoembolization in the treatment of middle and advanced liver cancer [J]. China Medicine and Pharmacy, 2020, 10(4):251-253,276 